\title{
Updated evaluation of clinicopathologic landscape in histopathologic subtypes of endometrial carcinoma with MMR gene mutation
}

\section{Aifen Wang ( $\sim$ aifen.wang@njucm.edu.cn )}

Zhangjiagang TCM Hospital Affiliated to Nanjing University of Chinese Medicine

\section{Weidong Hu}

ZhongNan Hospital of Wuhan University

\section{Qingfu Zhang}

the First Affiliated Hospital and College of Basic Medical Sciences of China Medical University

\section{Hongjiu Ren}

the First Affiliated Hospital and College of Basic Medical Sciences of China Medical University

\section{Ziyue Zhang}

University of California, San Diego

\section{Zihan Zhao}

University of California, Santa Barbara

Youguo Chen ( $\nabla$ chenyouguo@suda.edu.cn )

First Affiliated Hospital of Soochow University

\section{Research Article}

Keywords: Endometrioid endometrial cancer, MMR mutation, Uterine serous carcinoma, Prognosis

Posted Date: September 29th, 2020

DOI: https://doi.org/10.21203/rs.3.rs-83621/v1

License: (9) (i) This work is licensed under a Creative Commons Attribution 4.0 International License. Read Full License 


\section{Abstract}

Objective: This study aimed to identify an optimal screening strategy to detect mismatch repair (MMR) mutation for each histologic subtype of endometrial carcinoma.

Material and methods: We performed a comparative analysis of the demographic, clinical, pathologic, and molecular data for 562 patients from The Cancer Genome Atlas database, stratified by tumor histologic subtype.

Results: Molecular data, including MLH1, MLH3, PMS1, PMS2, MSH2, MSH3, MSH6, and EPCAM, was available for 562 patients, of which $162(28.8 \%)$ had tumors that were positive for MMR gene mutations. Of these tumors, the penetrate rate of grade 3 endometrioid endometrial carcinoma $(84 / 184,45.7 \%)$ was significantly higher than that of uterine serous carcinoma $(35 / 156,22.4 \%)(p<0.001)$, grade 2 endometrioid endometrial carcinoma $(26 / 129,20.2 \%)(p<0.001)$, and grade 1 endometrioid endometrial carcinoma $(17 / 93,18.3 \%)(p<0.001)$. Of 562 endometrial carcinomas, alterations in MSH2 $(\mathrm{n}=$ 55), MSH6 $(\mathrm{n}=54)$, and MSH3 $(\mathrm{n}=50)$ were the most frequent mutations. There were no differences in overall survival and progression-free interval (PFI) between MMR mutation carriers and nonmutation carriers ( $p>0.05$ ) except that PFI with MMR gene mutation was higher than with MMR proficiency in grade 3 endometrioid endometrial carcinoma $(p=0.014)$.

Conclusions: Grade 3 endometrioid endometrial carcinoma harbored more MMR mutations than grade 1 endometrioid endometrial carcinoma, grade 2 endometrioid endometrial carcinoma, and uterine serous carcinoma. Besides $M L H 1, M S H 2, M S H 6, P M S 2$, and EPCAM mutation, $M L H 3, M S H 3$, and PMS1 mutation may be necessary to be screened in patients with newly diagnosed endometrial carcinoma.

\section{Introduction}

Endometrial cancer is the most frequent malignancy of the female genital tract in women in advanced countries[1]. In the United States, there are more than 60,000 newly diagnosed cases and over 10,000 patients die from it every year. Mismatch repair (MMR) gene mutations are found in endometrial cancer and may be associated with Hereditary Nonpolyposis Colorectal Carcinoma or Lynch Syndrome. Notably, women with germline MMR mutation have a $43 \%$ risk of developing endometrial carcinoma [2]. Multiple factors, including family history, histological features, and age of diagnosis, have been utilized to demonstrate patients who could benefit from Lynch Syndrome screening. Prevalence studies have shown that the incidence of MMR mutations occurring in endometrial carcinoma range from $2 \%$ to $5.9 \%$ [3-9]. The most common "sentinel cancers" in patients with Lynch Syndrome are endometrial and colorectal cancers $[6,10-14]$. Universal screening of MMR mutations in newly diagnosed endometrial carcinoma was recommended by the Society of Gynecologic Oncology and National Comprehensive Cancer Network guideline (Version: February 2019).

There are numerous merits of detecting MMR deficiency among women with newly identified endometrial carcinoma. Firstly, surveillance testing for the patients with MMR mutation can detect Lynch Syndrome- 
associated cancers, like colorectal carcinoma and others. Second, relatives would have more chances of receiving surveillance testing, genetic counseling, as well as risk-reducing operations, including hysterectomy or colectomy. Finally, immunotherapy with PD-1/PD-L1 inhibitors have been a promising treatment for advanced/recurrent endometrial carcinoma with MMR mutations or microsatellite instability (MSI-H) $[7,15-18]$ and pembrolizumab was recommended by the National Comprehensive Cancer Network as a treatment option for recurrent MSI-H or MMR deficient endometrial cancer that has progressed after standard cytotoxic chemotherapy.

Currently, $M L H 1, M S H 2, M S H 6, P M S 2$, and EPCAM are routinely used in the screening strategy. $M L H 3$, $M S H 3$, and $P M S 1$ associated with Lynch Syndrome are not routinely tested. This study aims to add them to the current gene panel and compare demographic, clinicopathologic, and molecular characteristics of patients with endometrial cancer-based mutation status and stratified by histologic subtypes in order to provide evidence of optimal criteria for screening. These results may provide further evidence of the biology of MMR mutations, enhance clinical management of mutation carriers, and subsequently impact clinical trial design.

\section{Materials And Methods}

This study was approved by an institutional research ethics committee of Zhangjiagang TCM Hospital Affiliated to Nanjing University of Chinese Medicine (2019-023). The data released from the The Cancer Genome Atlas database did not require informed consent of patients because cancers are reportable diseases in every state in the United States.

\section{Study population}

We evaluated data from the publicly available National Cancer Institute The Cancer Genome Atlas EC database [http://www.cbioportal.org]. The final retrospective cohort was generated from 529 patients of the Pancancer Atlas and 548 patients from the Provisional database. All patients with endometrioid endometrial adenocarcinoma, or uterine serous carcinoma, or mixed endometroid and serous carcinoma or mixed clear and serous carcinoma were included in the study. After excluding duplicate patients from both databases $(n=515)$, the final cohort was comprised of 562 patients. We stratified the cohort into four groups of patients based on the MMR gene mutation status ( $M L H 1, M L H 3, P M S 1, P M S 2, M S H 2$, $M S H 3, M S H 6$, and EPCAM) and histology, including grade 1 endometroid endometrial carcinoma, grade 2 endometroid endometrial carcinoma, grade 3 endometroid endometrial carcinoma, and uterine serous carcinoma. Two experienced pathologists reviewed the pathologic diagnosis of all patients. We did not separate germline and somatic mutations. All patients were managed by National Comprehensive Cancer Network guidelines. The following variables were recorded for each case: age at diagnosis (categorized by $<50,50-69,70$ years old), mean age at the diagnosis, myometrial invasion (categorized $50 \%$ myometrial invasion, $<50 \%$ myometrial invasion), lymph node involvement, and clinical stage (categorized by FIGO IA, IB, II, III, IV). Definition of overall survival (OS) was the interval from the initial 
surgery to confirmation of death. Definition of progression-free interval (PFI) was the interval from the date of last chemotherapy or radiotherapy to the date of recurrence.

\section{Statistical Analysis}

Statistical analysis was performed using the Mann-Whitney test for continuous variables, and Chi-Square analysis or Fisher exact test for categorical variables, unless otherwise specified. The Student's $t$ test was performed in analyzing mean of body mass index and the age at diagnosis. Multivariate Cox proportional hazards models was used to analyze prognosis. OS or PFI was evaluated utilizing the log-rank test starting at the time of diagnosis, and the Kaplan-Meier method was used to generate survival curves. Statistical significance was evaluated at 0.05 level (exact 2-tailed). SPSS software (Version 23, IBM, USA) was applied in all calculations.

\section{Results}

Molecular data was available for 562 patients, of which $162(28.8 \%)$ had tumors that were positive for MMR gene mutations. Of these patients, 35 (22.4\%) were diagnosed with USC, 84 (45.7\%) with grade 3 endometroid endometrial carcinoma, $26(20.2 \%)$ with grade 2 endometroid endometrial carcinoma, and 17 (18.3\%) with grade 1 endometroid endometrial carcinoma (Table 1a). We found that MMR gene mutations occurred in grade 3 endometroid endometrial carcinoma significantly more frequently than in USC, grade 2 endometroid endometrial carcinoma, or grade 1 endometroid endometrial carcinoma (45.7\% vs $22.4 \%, p<0.001,45.7 \%$ vs $20.2 \%, p<0.001,45.7 \%$ vs $18.3 \%, p<0.001$, respectively, Table $1 \mathrm{a}$ ). We compared the distribution of tumors with MMR gene mutations per histology class, and there was no difference between USC (22.4\%) vs. grade 2 endometroid endometrial carcinoma $(20.2 \%, p=0.666)$, or grade 1 endometroid endometrial carcinoma $(18.3 \%, p=0.520)$.

Of the 562 endometrial carcinomas, alterations in MSH2 $(n=55), M S H 6(n=54)$, and MSH3 $(n=50)$ were the most frequent mutations. Among the USC, grade 3 endometroid endometrial carcinoma, grade 2 endometroid endometrial carcinoma, and grade 1 endometroid endometrial carcinoma, the most frequent alterations were $M S H 2, M S H 3, P M S 2$, and $M L H 3$, respectively (Table $1 \mathrm{~b}$ ). We analyzed $M L H 3, M S H, 3$ and PMS1 in this study since others have been well-studied. There were 30 patients with endometrial carcinoma with $M L H 3$ mutation, including 9 patients with only $M L H 3$ mutations and 21 women with MLH3 mutations cooccurrence with other MMR mutations (Table 1b). We found 50 patients with MSH3 mutation, including 17 patients with only $\mathrm{MSH} 3$ mutations and 33 women with $\mathrm{MSH} 3$ mutations cooccurrence with other MMR mutations (Table 1b). There were 20 patients with endometrial carcinoma with PMS1 mutation, including seven patients with only PMS1 mutations and 13 women with PMS1 mutations cooccurrence with other MMR mutations (Table 1b).

Among eight genes, two genes expressed simultaneously, including ten combinations; co-occurrence of three genes had six combinations; expression of four genes simultaneously presented eight combinations; five genes concurrently expressed two combinations; and cooccurrence of six genes had only 1 combination. (Table S1) 
Within grade 3 endometroid endometrial carcinoma, patients with MMR-mutated tumors were older and had lower body mass index than those with noncarriers ( $p=0.024, p=0.028$, respectively) (Table 2). Similarly, MMR deficient USC patients (Table 3 ) also had lower body mass index than MMR non carriers $(p=0.001)$. Lymph node metastasis was more frequent in patients with MMR mutations than noncarriers $(p=0.007)$. We found no differences in myometrial invasion, lymph node involvement, or FIGO stage between patients with MMR-mutated and nonmutated grade 3 endometroid endometrial carcinoma (Table 2). Similarly, patients with USC showed no association between age, body mass index, myometrial invasion, or FIGO stage and MMR status (Table 3 ). When considering MMR-mutated versus MMR nonmutated grade 2 endometroid endometrial carcinoma and grade 1 endometroid endometrial carcinoma, we did not find significant differences in age of diagnosis ( $p=0.426, p=0.332$, respectively), body mass index ( $p=0.668, p=0.464$, respectively), myometrial invasion ( $p=0.494, p=0.712$, respectively), FIGO stage ( $p=0.872, p=0.619$, respectively), or lymph node involvement $(p=0.072, p=0.203$, respectively) (Table S2, S3).

Favorable prognostic factors for all tumors included: myometrial invasion $<50 \%$ (OS and PFI), age $<50$ years old (OS), minimal invasive surgery (PFI), and lymph nodes negative (OS) were good prognosis factors (Table 4). We found no significant differences in OS and PFI between MMR mutation carriers and nonmutation carriers ( $p=0.647, p=0.350$, respectively) (Figure 1A, $1 \mathrm{~B}$ ). In our sub-group analysis, minimally invasive surgery was a good prognosis factor in grade 2 endometroid endometrial carcinoma; myometrial invasion < $50 \%$ was a good prognosis factor in grade 3 endometroid endometrial carcinoma; minimal invasive surgery, lymph node negative, and early stage were prognosis factors in USC (Table 4). In grade 3 endometroid endometrial carcinoma, PFI of MMR gene mutation was more favorable than with MMR proficiency ( $p=0.014$, Figure 1E); in contrast, OS was not affected by MMR status $(p=0.144,1 \mathrm{~F})$. MMR status was not associated with OS or PFI in patients with USC ( $p=0.560, p=0.891$; respectively, Figure 1G, $1 \mathrm{H})$, and grade 2 endometroid endometrial carcinoma ( $p=0.763, p=0.385$; Figure 1C, 1D). OS and PFI were not evaluated for grade 1 endometroid endometrial carcinoma due to limited cases with MMR mutation.

\section{Discussion}

To our knowledge, this is the first study to analyze clinicopathologic features and prognosis of endometrial carcinoma with MMR mutation by histologic subtypes, including uterine serous carcinoma which was considered to be strongly associated with TP53 and occasionally BRCA1/2 mutations. In addition, $M L H 3, M S H 3$, and $P M S 1$ genes were involved in this study. We identified that more MMR gene mutations occurred in grade 3 endometroid endometrial carcinoma than in grade 2 endometroid endometrial carcinoma, grade 1 endometroid endometrial carcinoma, or USC (mixed endometroid and serous carcinoma as well as mixed clear and endometroid carcinoma included) in our study. There were no significant differences in the penetrate of MMR mutations among USC, grade 2 endometroid endometrial carcinoma, and grade 1 endometroid endometrial carcinoma. Endometrial carcinomas have a diverse morphology histologically, with subtypes including endometrioid, clear cell, serous, and mixed (endometrioid and clear cell) carcinomas [19]. MLH1, MSH2, MSH6, PMS2, and EPCAM genes are most 
frequent and clinically related mutations in Lynch Syndrome [20,21]. One prospective study of over 3000 patients with Lynch Syndrome demonstrated that mutations occurred in $M L H 1, M S H 2$, and $M S H 6$, which total incidences of endometrial carcinoma (less than age 75 ) to be $43 \%, 57 \%$, and $46 \%$, respectively [2]. However, our study demonstrated that MSH2 $(\mathrm{n}=55), \mathrm{MSH} 3(\mathrm{n}=50)$, and MSH6 $(\mathrm{n}=54)$ mutations were the most frequent alterations among endometrial carcinomas. The total incidences of $M S H 2, M S H 3$, and MSH6 were much higher than previous literatures since we did not separate germline and somatic MMR mutation.

Limited data reported the role of $M L H 3, M S H 3$, and $P M S 1$ mutation in colorectal or endometrial cancers [22-25]. In this study, there were 30 patients with $M L H 3$ mutation, including 9 patients with only $M L H 3$ mutations. 50 patients were identified to harbor $\mathrm{MSH} 3$ mutation, including 17 patients with only $\mathrm{MSH} 3$ mutations. Of 20 patients with endometrial carcinoma with PMS1 mutation, seven patients had only PMS1 mutations. MLH3, MSH3, and PMS1 mutations may be necessary to screen MMR mutation in endometrial carcinoma.

Many studies demonstrated that prognosis of endometrial carcinoma with MMR mutation was more favorable than one without MMR mutation [20,21,26]. Sporadic or MMR proficient diseases are associated with older age of diagnosis, deeper tumor invasion, and more late-stage disease than patients with germline MMR mutations ( $M S H 2, M S H 6, P M S 2$, or $M H L 1$ absent $M H L 1$ methylation) [26]. Although MMR mutation carriers may have other Lynch Syndrome-associated tumors, they had demonstrated a better response rate to immune therapy than those with sporadic endometrial carcinoma. In contrast to previous reports, no differences were identified in OS or PFI ( $p=0.647, p=0.350$, respectively) between MMR-mutated and MMR-proficient tumors in this study. Sub-group analysis of MMR-mutated versus MMR-proficient tumors revealed that there were no differences in OS or PFI in grade 3 endometroid endometrial carcinoma, USC, and grade 2 endometroid endometrial carcinoma except that PFI in MMR mutated grade 3 endometrioid endometrial carcinomas was better than MMR mutation noncarriers.

Progesterone, which is commonly used in the management of patients with complex atypical hyperplasia or well-differentiated endometrial cancer, is not effective in patients with MMR mutation who are younger than 55 years of age. [27]. However, advanced Lynch Syndrome related cancers, including colorectal carcinoma, prostate cancer, and melanoma have promising therapeutic choices, such as immune checkpoint inhibitors, including programmed cell death ligand-1 (PDL-1) or PD-1 inhibitors. Surprisingly, comparison of $10 \%$ of MMR mutation noncarriers, PDL-1 expression is identified in $52.6 \%$ MMR mutation carriers with endometrial carcinoma; thus, PDL-1 inhibitor may be the best option for patients with MMR mutation carriers [28]. In another study, endometrial carcinomas with MMR mutations treated with immunotherapy were found to have a more favorable outcome [29]. A multicenter prospective study evaluated cancer detection among patients with MMR mutation through gynecologic and colonoscopy surveillance [30]. Besides MLH1, MSH2, MSH6, PMS2 and EPCAM mutations, MLH3, MSH3, and PMS1 deficiency may be screened in endometrial carcinoma to guide management. 
There are several limitations to this study. First, detailed information is unavailable concerning the patients' history, including family history, detailed chemotherapy, and radiotherapy. Second, the sample size of patients with MMR genetic defects could be relatively small, and much larger population-based studies may be necessary to detect survival differences. In addition, we did not stratify germline and somatic MMR mutation. In the future, we may focus on germline or somatic MMR mutation to investigate clinicopathologic feature and prognosis of endometrial carcinoma based on MMR mutations, including $M L H 3, M S H 3$, and PMS1 mutation.

\section{Conclusions}

Our study confirmed that endometrial carcinoma is strongly associated with MMR mutations, including USC histology. Grade 3 endometrioid endometrial cancer harbored more MMR mutations than Grade 1 endometrioid endometrial cancer, Grade 2 endometrioid endometrial cancer, and USC. MLH1, MSH2, $M S H 6, P M S 2$, and EPCAM deficiencies, $M L H 3, M S H 3, P M S 1$ mutations were common variants in endometrial cancers. Even though the prognosis of MMR mutation carriers was not more favorable than MMR proficient patients, immunotherapy is a promising new therapy for patients with MMR deficiencies, which was not likely used in our patients. Besides $M L H 1, M S H 2, M S H 6, P M S 2$, and EPCAM mutations, $M L H 3, M S H 3$, and $P M S 1$ mutations may be screened for Lynch Syndrome in patients with endometrial cancer to guide management and surveillance testing.

\section{Declarations}

\section{Conflict of interest}

Authors declared no conflicts of interests.

\section{Funding}

This study was not funded.

\section{Author contribution}

AW and WH analyzed and interpreted the data and was a major contributor in writing the manuscript; AW, YC and QZ interpreted the data; QZ and JR reviewed all pathologic images and conformed pathologic diagnosis; ZZ and ZZ abstracted the data; AW, YC and QZ designed the work and interpreted the data. All authors read and approved the final manuscript.

\section{References}

1. Siegel RL, Miller KD, Jemal A. Cancer statistics, 2020. CA Cancer J Clin. 2020;70(1):730.doi:10.3322/caac. 21590 
2. Moller P, Seppala TT, Bernstein I, Holinski-Feder E, Sala P, Gareth Evans D, et al. Cancer risk and survival in path_MMR carriers by gene and gender up to 75 years of age: a report from the Prospective Lynch Syndrome Database. Gut. 2018;67(7):1306-16.doi:10.1136/gutjnl-2017-314057

3. Mills AM, Longacre TA. Lynch Syndrome Screening in the Gynecologic Tract: Current State of the Art. Am J Surg Pathol. 2016;40(4):e35-44.doi:10.1097/PAS.0000000000000608

4. Lu KH, Ring KL. One size may not fit all: the debate of universal tumor testing for Lynch syndrome. Gynecol Oncol. 2015;137(1):2-3.doi:10.1016/j.ygyno.2015.03.011

5. Lin DI, Hecht JL. Targeted Screening With Combined Age- and Morphology-Based Criteria Enriches Detection of Lynch Syndrome in Endometrial Cancer. Int J Surg Pathol. 2016;24(4):297305.doi:10.1177/1066896916629782

6. Hampel H, Frankel W, Panescu J, Lockman J, Sotamaa K, Fix D, et al. Screening for Lynch syndrome (hereditary nonpolyposis colorectal cancer) among endometrial cancer patients. Cancer Res. 2006;66(15):7810-7.doi:10.1158/0008-5472.CAN-06-1114

7. Ollikainen M, Abdel-Rahman WM, Moisio AL, Lindroos A, Kariola R, Jarvela I, et al. Molecular analysis of familial endometrial carcinoma: a manifestation of hereditary nonpolyposis colorectal cancer or a separate syndrome? J Clin Oncol. 2005;23(21):4609-16.doi:10.1200/JC0.2005.06.055

8. Egoavil C, Alenda C, Castillejo A, Paya A, Peiro G, Sanchez-Heras AB, et al. Prevalence of Lynch syndrome among patients with newly diagnosed endometrial cancers. PLoS One. 2013;8(11):e79737.doi:10.1371/journal.pone.0079737

9. Dillon JL, Gonzalez JL, DeMars L, Bloch KJ, Tafe LJ. Universal screening for Lynch syndrome in endometrial cancers: frequency of germline mutations and identification of patients with Lynch-like syndrome. Hum Pathol. 2017;70:121-8.doi:10.1016/j.humpath.2017.10.022

10. Lynch HT, de la Chapelle A. Hereditary colorectal cancer. N Engl J Med. 2003;348(10):91932.doi:10.1056/NEJMra012242

11. Resnick KE, Hampel H, Fishel R, Cohn DE. Current and emerging trends in Lynch syndrome identification in women with endometrial cancer. Gynecol Oncol. 2009;114(1):128-

34.doi:10.1016/j.ygyno.2009.03.003

12. Lu KH, Dinh M, Kohlmann W, Watson P, Green J, Syngal S, et al. Gynecologic cancer as a "sentinel cancer" for women with hereditary nonpolyposis colorectal cancer syndrome. Obstet Gynecol. 2005;105(3):569-74.doi:10.1097/01.AOG.0000154885.44002.ae

13. Aaltonen LA, Salovaara R, Kristo P, Canzian F, Hemminki A, Peltomaki P, et al. Incidence of hereditary nonpolyposis colorectal cancer and the feasibility of molecular screening for the disease. $\mathrm{N}$ Engl J Med. 1998;338(21):1481-7.doi:10.1056/NEJM199805213382101

14. Hampel H, Frankel WL, Martin E, Arnold M, Khanduja K, Kuebler P, et al. Feasibility of screening for Lynch syndrome among patients with colorectal cancer. J Clin Oncol. 2008;26(35):57838.doi:10.1200/JC0.2008.17.5950

15. Lynch HT, Lynch PM, Lanspa SJ, Snyder CL, Lynch JF, Boland CR. Review of the Lynch syndrome: history, molecular genetics, screening, differential diagnosis, and medicolegal ramifications. Clin 
Genet. 2009;76(1):1-18.doi:10.1111/j.1399-0004.2009.01230.x

16. Lancaster JM, Powell CB, Kauff ND, Cass I, Chen LM, Lu KH, et al. Society of Gynecologic Oncologists Education Committee statement on risk assessment for inherited gynecologic cancer predispositions. Gynecol Oncol. 2007;107(2):159-62.doi:10.1016/j.ygyno.2007.09.031

17. Lindor NM, Petersen GM, Hadley DW, Kinney AY, Miesfeldt S, Lu KH, et al. Recommendations for the care of individuals with an inherited predisposition to Lynch syndrome: a systematic review. JAMA. 2006;296(12):1507-17.doi:10.1001/jama.296.12.1507

18. Resnick K, Straughn JM, Jr., Backes F, Hampel H, Matthews KS, Cohn DE. Lynch syndrome screening strategies among newly diagnosed endometrial cancer patients. Obstet Gynecol. 2009;114(3):5306.doi:10.1097/AOG.0b013e3181b11ecc

19. Carcangiu ML, Radice P, Casalini P, Bertario L, Merola M, Sala P. Lynch syndrome-related endometrial carcinomas show a high frequency of nonendometrioid types and of high FIGO grade endometrioid types. Int J Surg Pathol. 2010;18(1):21-6.doi:10.1177/1066896909332117

20. Rhyu MS. Molecular mechanisms underlying hereditary nonpolyposis colorectal carcinoma. J Natl Cancer Inst. 1996;88(5):240-51

21. Miyaki M, Konishi M, Tanaka K, Kikuchi-Yanoshita R, Muraoka M, Yasuno M, et al. Germline mutation of MSH6 as the cause of hereditary nonpolyposis colorectal cancer. Nat Genet. 1997;17(3):2712.doi:10.1038/ng1197-271

22. Korhonen MK, Vuorenmaa E, Nystrom M. The first functional study of MLH3 mutations found in cancer patients. Genes Chromosomes Cancer. 2008;47(9):803-9.doi:10.1002/gcc.20581

23. Wu Y, Berends MJ, Sijmons RH, Mensink RG, Verlind E, Kooi KA, et al. A role for MLH3 in hereditary nonpolyposis colorectal cancer. Nat Genet. 2001;29(2):137-8.doi:10.1038/ng1001-137

24. Risinger JI, Umar A, Boyd J, Berchuck A, Kunkel TA, Barrett JC. Mutation of MSH3 in endometrial cancer and evidence for its functional role in heteroduplex repair. Nat Genet. 1996;14(1):102-

5.doi:10.1038/ng0996-102

25. Hienonen T, Laiho P, Salovaara R, Mecklin JP, Jarvinen H, Sistonen P, et al. Little evidence for involvement of MLH3 in colorectal cancer predisposition. Int J Cancer. 2003;106(2):2926.doi:10.1002/ijc. 11218

26. Shikama A, Minaguchi T, Matsumoto K, Akiyama-Abe A, Nakamura Y, Michikami $H$, et al. Clinicopathologic implications of DNA mismatch repair status in endometrial carcinomas. Gynecol Oncol. 2016;140(2):226-33.doi:10.1016/j.ygyno.2015.11.032

27. Zakhour M, Cohen JG, Gibson A, Walts AE, Karimian B, Baltayan A, et al. Abnormal mismatch repair and other clinicopathologic predictors of poor response to progestin treatment in young women with endometrial complex atypical hyperplasia and well-differentiated endometrial adenocarcinoma: a consecutive case series. BJOG. 2017;124(10):1576-83.doi:10.1111/1471-0528.14491

28. Sloan EA, Ring KL, Willis BC, Modesitt SC, Mills AM. PD-L1 Expression in Mismatch Repair-deficient Endometrial Carcinomas, Including Lynch Syndrome-associated and MLH1 Promoter 
Hypermethylated Tumors. Am J Surg Pathol. 2017;41(3):326-

33.doi:10.1097/PAS.0000000000000783

29. Moller P, Seppala T, Bernstein I, Holinski-Feder E, Sala P, Evans DG, et al. Cancer incidence and survival in Lynch syndrome patients receiving colonoscopic and gynaecological surveillance: first report from the prospective Lynch syndrome database. Gut. 2017;66(3):464-72.doi:10.1136/gutjnl2015-309675

30. Moller P, Seppala T, Bernstein I, Holinski-Feder E, Sala P, Evans DG, et al. Incidence of and survival after subsequent cancers in carriers of pathogenic MMR variants with previous cancer: a report from the prospective Lynch syndrome database. Gut. 2017;66(9):1657-64.doi:10.1136/gutjnl-2016-311403

\section{Tables}

Table 1A: MMR mutation distribution in EEC subtypes and USC

\begin{tabular}{|lll|}
\hline EC Subtypes & MMR Mutation status & No. (\%) \\
\hline EC & MMR Mutation & $162(28.8)$ \\
\cline { 2 - 3 } EEC G1 & MMR Nonmutation & $400(71.2)$ \\
& MMR Mutation & $17(18.3)$ \\
\cline { 2 - 3 } EEC G2 & MMR Nonmutation & $76(81.7)$ \\
\hline EEC G3 & MMR Mutation & $26(20.2)$ \\
\cline { 2 - 3 } & MMR Nonmutation & $103(79.8)$ \\
\hline USC & MMR Mutation & $84(45.7)$ \\
& MMR Mutation & $100(54.3)$ \\
\cline { 2 - 3 } & MMR Nonmutation & $121(77.6)$ \\
\hline
\end{tabular}

Abbreviations: EC, endometrial cancer; EEC G1, grade 1 endometroid endometrial carcinoma; EEC G2, grade 2 endometroid endometrial carcinoma; EEC G3, grade 3 endometroid endometrial carcinoma; USC, uterine serous carcinoma.

Table 1B: MMR mutation distribution among the endometrial carcinoma 


\begin{tabular}{|llllll|}
\hline MMR & USC & EEC G3 & EEC G2 & EEC G1 & Total \\
\hline MLH1 & $4(2.56 \%)$ & $25(13.59 \%)$ & $6(4.65 \%)$ & $3(3.22 \%)$ & $38(6.76 \%)$ \\
\hline MLH3 & $6(3.85 \%)$ & $14(7.61 \%)$ & $6(4.65 \%)$ & $4(4.30 \%)$ & $30(5.34 \%)$ \\
\hline MSH2 & $15(9.61 \%)$ & $31(16.85 \%)$ & $6(4.65 \%)$ & $3(3.22 \%)$ & $55(9.79 \%)$ \\
\hline MSH3 & $6(3.85 \%)$ & $35(19.02 \%)$ & $7(5.43 \%)$ & $2(2.15 \%)$ & $50(8.90 \%)$ \\
\hline MSH6 & $13(8.33 \%)$ & $31(16.85 \%)$ & $8(6.2 \%)$ & $3(3.22 \%)$ & $54(9.61 \%)$ \\
\hline PMS1 & $4(2.56 \%)$ & $13(7.1 \%)$ & $1(0.77 \%)$ & $2(2.15 \%)$ & $20(3.56 \%)$ \\
\hline PMS2 & $2(1.28 \%)$ & $23(12.5 \%)$ & $8(6.20 \%)$ & $4(4.30 \%)$ & $37(6.58 \%)$ \\
\hline EPCAM & $6(3.84 \%)$ & $7(0.54 \%)$ & $3(2.32 \%)$ & $1(0.11 \%)$ & $17(3.2 \%)$ \\
\hline
\end{tabular}

Abbreviations: $\mathrm{EC}$, endometrial cancer; $\mathrm{EEC} \mathrm{G1}$, grade 1 endometroid endometrial carcinoma; $\mathrm{EEC} \mathrm{G} 2$, grade 2 endometroid endometrial carcinoma; EEC G3, grade 3 endometroid endometrial carcinoma, USC, uterine serous carcinoma.

Table 2هComparison of clinicopathologic features of EEC3 with MMR mutation vs. MMR nonmutation 


\begin{tabular}{|c|c|c|c|}
\hline Patient status & MMR mutation & MMR nonmutation & $P$ value \\
\hline & $\mathrm{n}(\%)$ & $\mathrm{n}(\%)$ & \\
\hline EEC3 & 84 & 100 & \\
\hline Body mass index & & & 0.102 \\
\hline$<25$ & $22(26.2)$ & $13(13)$ & \\
\hline $25-29$ & $15(17.9)$ & $16(16)$ & \\
\hline 30 & $38(45.2)$ & $65(65)$ & \\
\hline Unknown & $9(10.7)$ & $6(6)$ & \\
\hline Mean \pm SD & $31.4 \pm 9.2$ & $34.5 \pm 8.7$ & 0.028 \\
\hline \multicolumn{4}{|l|}{ Age (years) } \\
\hline Mean \pm SD & $60.9 \pm 13.0$ & $64.9 \pm 10.6$ & 0.024 \\
\hline 70 & $19(22.6)$ & $32(32)$ & 0.024 \\
\hline $50-69$ & $48(57.1)$ & $60(60)$ & \\
\hline$<50$ & $14(16.7)$ & $7(7)$ & \\
\hline Unknown & $3(3.6)$ & $1(1)$ & \\
\hline \multicolumn{4}{|l|}{ Tumor invasion } \\
\hline $50 \%$ & $47(56.0)$ & $50(50)$ & 0.295 \\
\hline$<50 \%$ & $36(42.9)$ & $44(44)$ & \\
\hline Unknown & $1(1.2)$ & $6(6)$ & \\
\hline \multicolumn{4}{|l|}{ Lymph node involved } \\
\hline+ & $15(17.8)$ & $16(16)$ & 0.843 \\
\hline- & $60(71.4)$ & $70(70)$ & \\
\hline No Lymphadenectomy & $8(9.5)$ & $6(6)$ & \\
\hline Unknown & $1(1.2)$ & $4(4)$ & \\
\hline FIGO stage & & & 0.534 \\
\hline IA & $31(36.9)$ & $33(33)$ & \\
\hline IB & $22(26.2)$ & $29(29)$ & \\
\hline II & $6(7.1)$ & $8(8)$ & \\
\hline III & $23(27.4)$ & $20(20)$ & \\
\hline
\end{tabular}




IV $\quad 2(2.4) \quad 10(10)$

Abbreviations: EEC G3, grade 3 endometroid endometrial carcinoma.

Table 3囚 Comparison of clinicopathologic features of USC with MMR mutation vs. MMR nonmutation 


\begin{tabular}{|c|c|c|c|}
\hline Patient status & MMR deficient & MMR proficient & $P$ value \\
\hline & $\mathrm{n}(\%)$ & $\mathrm{n}(\%)$ & \\
\hline USC & 35 & 121 & 0.245 \\
\hline Body mass index & & & 0.001 \\
\hline$<25$ & $6(17.1)$ & $36(29.8)$ & \\
\hline $25-29$ & $7(20)$ & $26(21.5)$ & \\
\hline \multirow[t]{2}{*}{30} & $21(60)$ & $46(38)$ & \\
\hline & $2(5.7)$ & $13(10.7)$ & \\
\hline Mean \pm SD & $29.8 \pm 7.4$ & $31.8 \pm 8.7$ & 0.193 \\
\hline \multicolumn{4}{|l|}{ Age (years) } \\
\hline Mean \pm SD & $66.3 \pm 10.2$ & $68.7 \pm 8.3$ & 0.173 \\
\hline 70 & $13(37.1)$ & $52(43)$ & 0.254 \\
\hline $50-69$ & $19(54.3)$ & $69(57)$ & \\
\hline$<50$ & $3(8.6)$ & 0 & \\
\hline \multicolumn{4}{|l|}{ Tumor invasion } \\
\hline $50 \%$ & $18(51.4)$ & $44(36.4)$ & 0.120 \\
\hline$<50 \%$ & $17(48.6)$ & $77(63.6)$ & \\
\hline \multicolumn{4}{|l|}{ Lymph nodes involved } \\
\hline+ & $3(8.6)$ & $36(29.8)$ & 0.007 \\
\hline- & $20(57.1)$ & $59(48.8)$ & \\
\hline No Lymphadenectomy & $4(11.4)$ & $9(7.4)$ & \\
\hline Unknown & $8(22.9)$ & $15(12.4)$ & \\
\hline FIGO stage & & & 0.297 \\
\hline IA & $9(25.7)$ & $29(24)$ & \\
\hline IB & $6(17.1)$ & $18(14.9)$ & \\
\hline II & $7(20)$ & $11(9.1)$ & \\
\hline III & $10(28.6)$ & $47(38.8)$ & \\
\hline IV & $3(8.6)$ & $16(13.2)$ & \\
\hline
\end{tabular}

Abbreviations: USC, uterine serous carcinoma. 
Table 4: Relative risk of OS and PFI in patients with EC (Multivariate Cox hazards model).

\begin{tabular}{|c|c|c|c|c|}
\hline & \multicolumn{4}{|c|}{ All patients $(N=562)$} \\
\hline & OS $(406 / 562)$ & & PFI (390/562) & \\
\hline Variable name & $\mathrm{HR}[95 \% \mathrm{Cl}]$ & $P$ & $\mathrm{HR}[95 \% \mathrm{Cl}]$ & $P$ \\
\hline Myometrial invasion $<50 \%$ vs. $50 \%$ & $0.01[0.00-0.41]$ & $<0.001$ & $0.05[0.01-0.46]$ & 0.008 \\
\hline Lymph node - vs. Lymph node + & $0.40[0.19-0.86]$ & 0.019 & - & - \\
\hline Age range from $50-69$ y vs. $<50$ y & $3.95[1.11-14.06]$ & 0.034 & - & - \\
\hline Open Surgery vs. Minimally Invasive & - & - & $2.19[1.28-3.73]$ & 0.004 \\
\hline
\end{tabular}

Abbreviations, OS, overall survival; PFI, progression-free interval; EC, endometrial cancer

\section{Figures}



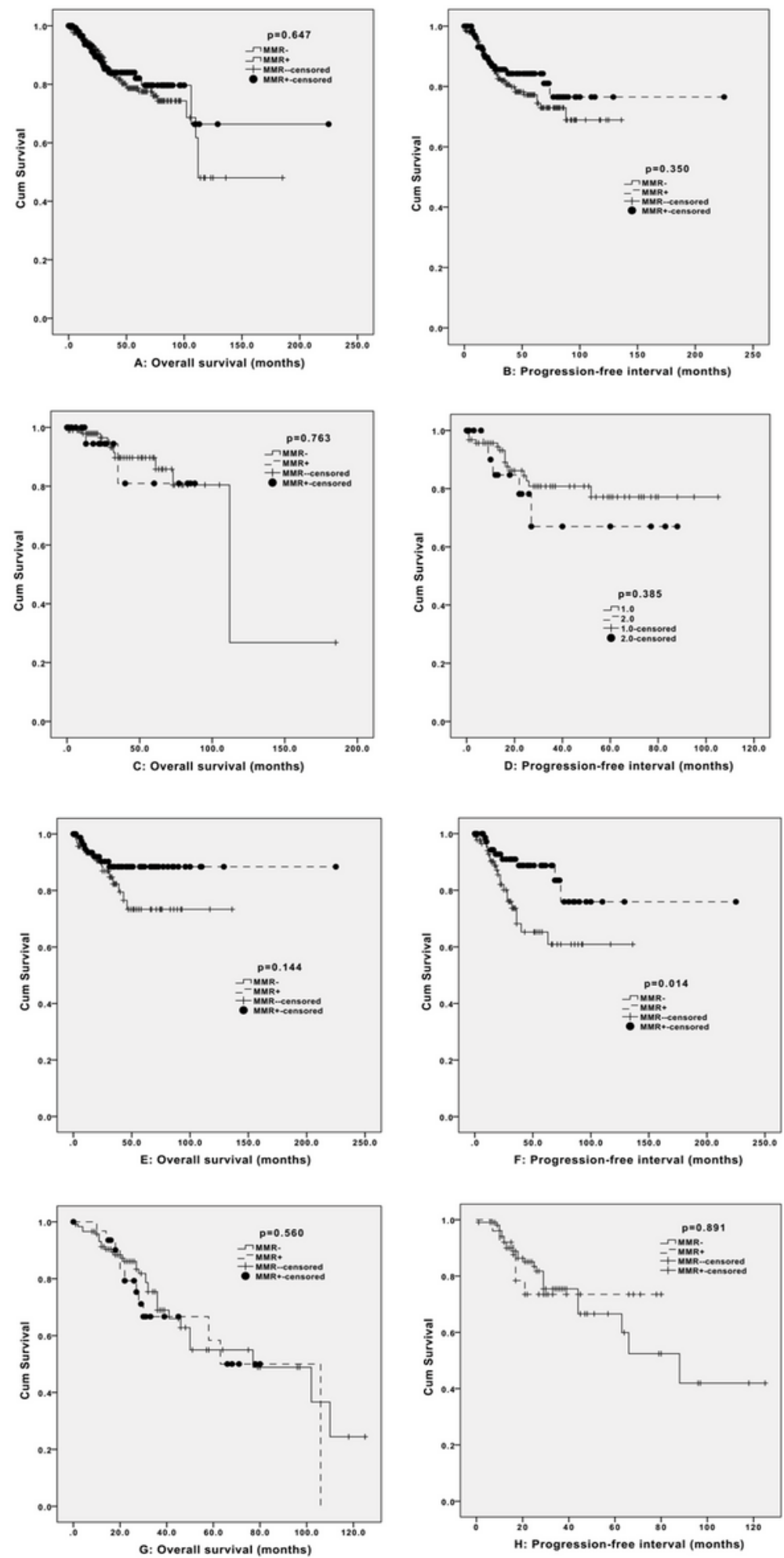

Figure 1

Overall survival and progression-free interval of endometrial carcinoma subtypes

\section{Supplementary Files}

This is a list of supplementary files associated with this preprint. Click to download. 
- SupplementalTables.docx

Page 17/17 